
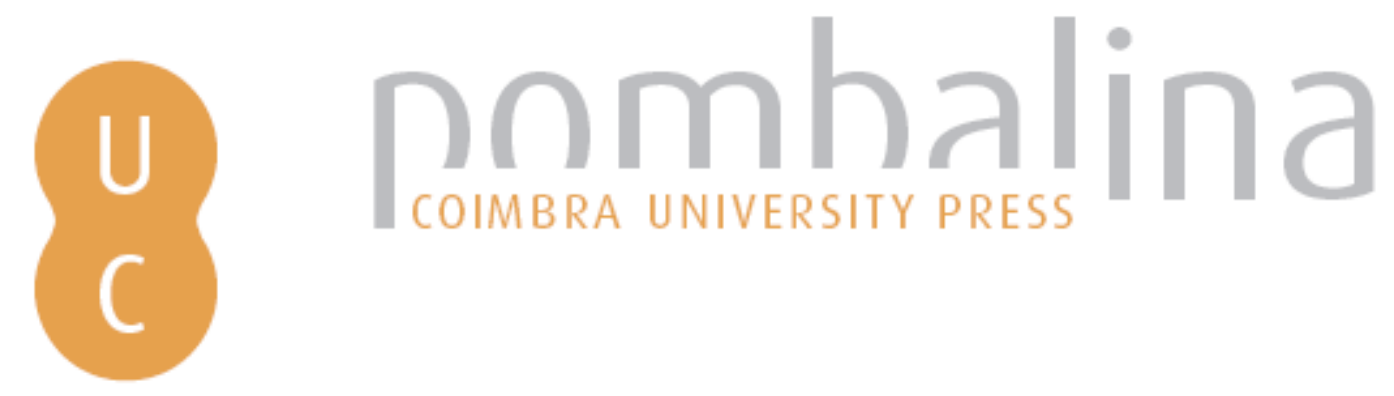

\title{
«Et il fuoco fa che la pignata mandi sopra la schiuma»: quando la lussuria sta in cucina? Santi francescani italo-lusitani e disciplina sociale tra medioevo e prima etá moderna
}

Autor(es): $\quad$ Nestola, Paola

Publicado por: Imprensa da Universidade de Coimbra

URL persistente:

URI:http://hdl.handle.net/10316.2/45236

DOI:

DOI:https://doi.org/10.14195/978-989-26-1721-3_9

Accessed : $\quad$ 26-Apr-2023 14:03:50

A navegação consulta e descarregamento dos títulos inseridos nas Bibliotecas Digitais UC Digitalis, UC Pombalina e UC Impactum, pressupõem a aceitação plena e sem reservas dos Termos e Condições de Uso destas Bibliotecas Digitais, disponíveis em https://digitalis.uc.pt/pt-pt/termos.

Conforme exposto nos referidos Termos e Condições de Uso, o descarregamento de títulos de acesso restrito requer uma licença válida de autorização devendo o utilizador aceder ao(s) documento(s) a partir de um endereço de IP da instituição detentora da supramencionada licença.

Ao utilizador é apenas permitido o descarregamento para uso pessoal, pelo que o emprego do(s) título(s) descarregado(s) para outro fim, designadamente comercial, carece de autorização do respetivo autor ou editor da obra.

Na medida em que todas as obras da UC Digitalis se encontram protegidas pelo Código do Direito de Autor e Direitos Conexos e demais legislação aplicável, toda a cópia, parcial ou total, deste documento, nos casos em que é legalmente admitida, deverá conter ou fazer-se acompanhar por este aviso.

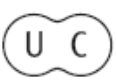


Carmen Soares

Gilene da Silva Gomes Ribeiro

(coords.)

\section{Mesas \\ LUSO-BRASILEIRAS}

\section{ALIMENTAÇÃO, SAÚdE \& GULTURA}

\section{VOLUME I}

IMPRENSA DA UNIVERSIDADE DE COIMBRA COIMBRA UNIVERSITY PRESS

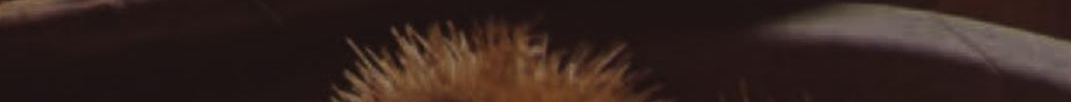




\title{
«ET IL FUOCO FA CHE LA PIGNATA MANDI SOPRA LA SCHIUMA": QUANDO LA LUSSURIA STA IN CUCINA? SANTI FRANCESCANI ITALO-LUSITANI E DISCIPLINA SOCIALE TRA MEDIOEVO E PRIMA ETÁ MODERNA*
}

\author{
(When lust is in the kitchen? Franciscan saints and social \\ discipline between the Middle Ages and Early Modern period)
}

\author{
Paola Nestola \\ Centro de História da Sociedade e da Cultura \\ Universidade de Coimbra \\ (nestolap@gmail.com)
}

Riassunto: Questo testo intende analizzare il tema della lussuria e delle proposte di disciplina sociale nell'ambito della confessione sacramentale nella prima metà del '600, partendo dal linguaggio metaforico usato dal frate italiano fra Giuseppe da Copertino/José de Cupertino (1603-1663). La metafora culinaria del frate francescano (canonizzato nel XVIII secolo) riprende le forti esortazioni moraleggianti usate dal correligionario S. Antonio di Lisbona (1195-1231). Anche il dotto e popolare predicatore portoghese faceva uso di simboliche immagini per raffigurare i vizi capitali. Fonti descrittive come processi di canonizzazione di San Giuseppe da Copertino, agiografie, sermoni di Sant'Antonio, testi legislativi sinodali e documenti iconografici sono alla base di questo percorso analitico centrato su alcuni territori dell'orbe cattolico, come la penisola italiana meridionale o il Portogallo.

Parole chiavi: lussuria, metafora culinaria, confessione sacramentale, disciplina sociale, agiografie francescane, S. Giuseppe da Copertino, S. Antonio di Lisbona, XVII secolo, Puglia, Portogallo.

Aвstract: This text intends to analyze the theme of lust and proposals of social discipline within the sacramental confession in the first half of $17^{\text {th }}$ century, based on the metaphorical language used by the Italian friar Joseph of Cupertino (1603-1663). The culinary metaphor of the Franciscan friar (canonized in the $18^{\text {th }}$ century) takes strong moralizing exhortations used by coreligionist St. Anthony of Lisbon (1195-1231). Even the erudite and popular Portuguese preacher made use of symbolic images to represent the seven deadly sins. Descriptive sources as the processes of canonization of St. Joseph of Cupertino, St. Antony's sermons, legislative texts of Episcopal synods and iconographic documents are the basis of

* Questo contributo è svolto nell'ambito della borsa di post-dottorato Fundação para a Ciência e a Tecnologia de Portugal (SFRH/BPD/62887/2009). Una versione piú estesa di questo studio fa parte del volume: Paola Nestola, San Giuseppe da Copertino: dall'estrema Puglia al Portogallo, Ed. Grifo-CHSC, Lecce-Coimbra, 2016. 
this analytical path, centered on some peripheral areas of the Catholic world, like the South of Italy or Portugal.

KEYWORDs: lust, culinary metaphor, sacramental confession, social discipline, Franciscan hagiography, St. Joseph of Cupertino, St. Antony of Lisbon, $17^{\text {th }}$ C., Apulia, Portugal.

Piacere gastronomico e piacere sessuale costituiscono un antico topos letterario oltre che iconografico, trattato molte volte fin dall'epoca medievale, associando e correlando vizi come gola e lussuria ${ }^{1}$. Di quest'ultimo vizio carnale il popolare predicatore lisboneta sant'Antonio di Padova (1195-1231) illustrava l'etimologia nel sermone della II domenica dopo l'ottava dell'Epifania, con la seguente suggestiva immagine moraleggiante: "Luxuria dicitur a luxu cibi et potus, quorum affluentia caro viva et petulans luxuriantur. Tali vita omnis vivens non iustificabitur in conspectu tuo, immo condemnabitur" ${ }^{2}$.

Forse l'incisivo ammonimento del dotto santo medievale, diffuso in una delle versioni a stampa dei suoi sermoni latini disponibili nelle biblioteche di Terra d'Otranto (nel sud della penisola italiana) ${ }^{3}$, oppure predicato con un linguaggio più semplice e accessibile per il largo pubblico ${ }^{4}$, avrà influenzato in qualche misura sia la metafora culinaria che avvia il titolo di questo contributo, sia il regime alimentare di chi la proferì. La proposta analitica, difatti, intende analizzare una espressione figurata usata dal francescano italiano Giuseppe da Copertino/ José de Cupertino (1603-1663), asceso agli onori degli altari nel corso del Settecento ${ }^{5}$. Il linguaggio culinario usato dal religioso pugliese - correligionario di Sant'Antonio - è datato alla prima metà del ' 600 , inserendosi in un contesto ideologico e socio-culturale in cui le questioni sessuali costituivano argomento preminente nell'ambito della confessione sacramentale ${ }^{6}$. È possibile avanzare questo percorso soprattutto attraverso fonti agiografiche come i processi di canonizzazione del santo pugliese, considerato un esempio significativo della mistica seicentesca.

1 Montanari 2012: 196-197; Baschet, 2000: 225-260; Belting 2005.

2 S. Antonii Patavinii 1979: vol. II, 587.

3 De Luca 2000: 119, 171, 208, 209.

4 Rusconi 2002: 105-160. Per periodi successivi, laddove viene sempre più assoggettata al controllo vescovile: Idem 1981: 951-1035. Per il territorio di Terra d'Otranto, riguardo al passaggio dalla predicazione francescana a quella soggetta al controllo vescovile: Nestola 2008: 207-231. Centrato soprattutto sullo spazio portoghese: Marques, 2000: vol. II, 393-447; Paiva 2009: 7-42; Idem 2013: 217-258, 218-219.

${ }^{5}$ Si rimanda alle rispettive voci: Giuseppe da Copertino/José de Cupertino: Del Re 1965: 1300-1303; Campos 1990: 87; Alkofer 2006: 820-821.

6 Ginzburg 1986: 133-157; Prosperi 1996: 476-542; Romeo 1998; De Boer 2004: 21-34; Gouveia 2011: 35-43; Gouveia 2015. 
Già dal 1993 il contributo di Christian Renoux ha evidenziato le molteplici potenzialità di questa eterogenea tipologia documentale per gli studi di storia delle mentalità, così come per altri cantieri storiografici tra cui la storia della vita quotidiana nei conventi ${ }^{7}$. Nel caso specifico, relativo al frate copertinese, potremmo dire che resta ancora molto da fare, avendo a disposizione un articolato corpus manoscritto o a stampa sia periferico che centrale, reso sempre più facilmente fruibile agli studiosi ${ }^{8}$.

Il culto verso questo modello di santità nativo della Puglia e reclutato nell'ordine francescano, d'altra parte, ebbe una diffusione molto estesa nell'orbe cattolico, tra cui il Portogallo, territorio di origine di Sant'Antonio. A partire dalla beatificazione del frate avvenuta nel 1753, la devozione verso il religioso oriundo di Copertino venne favorita dallo stesso ordine serafico nell'estrema penisola iberica 9 . In pratica dal luglio del 1755 , la proposta venne avanzata al pubblico di Lisbona dalla radicata istituzione regolare $^{10}$, secondo modelli che si rifacevano sia al fondatore san Francesco d'Assisi, sia a quelli del grande taumaturgo lisboneta.

Uno dei punti di congiunzione con la tradizione medioevale era lo speciale rapporto instaurato dal neo-beato con gli animali. Diversamente dal modello dei santi duecenteschi, di cui gli esempi iconografici esaltavano le doti oratorie del miracolo della predicazione ai pesci o del discorso agli uccelli, o della conversione del lupo, il beato Jozé de Copertino veniva presentato al pubblico della capitale portoghese intento ad alimentare "as aves", di cui parlava fr. Manoel de S. Dâmaso nelle sue Memorias ${ }^{11}$. In tale breve notizia che descriveva gli animali dell'aria pronti a nutrirsi dalle mani del religioso asceso al titolo di beato, con una attenta simbologia si alludeva verosimilmente alla caratteristica identificativa delle estasi e dei voli josefini, cosí come ad altre virtù eroiche che caratterizzavano quel nuovo modello di santità. Una innocenza "candida" e "naturale" a detta del confratello e accademico della Real Accademia di Storia fra Manoel, per cui anche gli uccelli lo trattavano "familiarmente". Attraverso questo peculiare modello agiografico francescano che condensava pure la continenza e l'astensione da certi cibi come la carne ${ }^{12}$, si invertivano alcune delle forme principali di relazione tra mondi diversi: sia quella secondo cui la carne dei volatili costituiva una fonte di alimentazione per gli umani; sia quella per cui la

\footnotetext{
7 Renoux 1993: 177-217.

8 Spedicato 2015: 315-326; Nestola 2015: 327-341.

9 Nestola 2013: 95-118.

10 Montes Moreira 2000: 273-280; Andrade 2010: 158-169; Silva 2010: 169-173; Teixeira

11 Dâmaso 1755: 7-8.

12 Gentilcore 2015: 103.
} 2015: 7-53. 
caccia era uno dei passatempi tanto dell'aristocrazia quanto di classi meno agiate $^{13}$. Si tratta di una tappa importante del percorso di costruzione e di identificazione nell'immaginario collettivo lusitano di quel nuovo schema agiografico applicato ad un religioso forestiero. Un momento di promozione del nuovo culto nella penisola atlantica, ma che contribuisce a definire meglio quello costruito nel territorio di origine del frate nativo della provincia del Mezzogiorno napoletano.

A tutt'oggi nello spazio lusitano le fonti agiografiche josefine sono costituite da documenti editi nella prima metà del Settecento, come la breve ma incisiva Memoria redatta dal confratello e accademico della Real Accademia di Storia, fra Manoel de S. Dâmaso. Una tipologia documentale diversa rispetto a quanto rimane nella penisola italiana dove, accanto a testi a stampa, sono disponibili pure i processi manoscritti, imbastiti subito dopo la morte del frate nel 1663, e necessari per avviare il processo di canonizzazione di fra Giuseppe. Proprio attraverso l'analisi di questo eterogeneo corpus agiografico è possibile abbordare il tema della lussuria e delle proposte disciplinari suggerite dal frate italiano: un discorso metaforico che, per certi aspetti, sembra riecheggiare le forti tinte moraleggianti usate dal predicatore portoghese correligionario S. Antonio, il quale, a sua volta, faceva uso di simboliche immagini per raffigurare i vizi capitali ${ }^{14}$.

Una delle più diffuse metafore antoniane adoperate per rappresentare la lussuria è proprio quella in cui coloro che si abbandonano a questo vizio carnale vengono definiti come agitati dagli spiriti immondi, e paragonati ad una pentola posta sul fuoco, secondo quanto viene riportato nel sermone della IV domenica dopo Pasqua: "Faciunt enim sicut olla posita super ignem, quae spumam emittit. Olla est cor peccatoris, in qua est aqua concupiscientia carnalis, cui supponitur ignis diabolicae suggestionis, et sic despumat luxuriam suae confusionis" 15 .

Questa complessa simbologia è stata oggetto di alcuni studi da parte della storiografia antoniana, a partire dal contributo di Gianfranco Gardin del 1982, al quale è seguita una specifica voce del Dizionario Antoniano ${ }^{16}$. In questi percorsi gli autori hanno puntato sui termini e sulle immagini usate dal dotto predicatore per indicare comportamenti sessuali o immorali diversi, articolati come un sistema di vizi di cui il maledictus ternarius (lussuria, superbia, avarizia) costituiva uno dei punti centrali, e a cui si poteva controbattere con forme penitenziali specifiche, quali penitenze corporali o spirituali (digiuni, ma anche attraverso la preghiera, la contemplazione, la confessione, etc.). Spunti e segnalazioni metodologiche che dovrebbero trovare un ulteriore

\footnotetext{
13 Drumond Braga 2015: 13-31.

${ }_{14}$ Cargnoni 2002: 918-986; Gardin 1982: 701-719; Cargnoni 2002: 431-448.

15 S. Antonii Patavinii 1979: vol. I, 319; Santo António de Lisboa, 1970: vol. I, 249.

${ }^{16} \mathrm{Cf}$. note 2 e 15.
} 
riscontro con il linguaggio usato dal francescano pugliese di epoca moderna, con il suo modus vivendi, e con i diversi universi socio-culturali che deposero ai processi come quello di Nardò, contribuendo alla definizione del suo modello agiografico. Finora difatti è stata privilegiata l'ottica ricollegabile a S. Francesco d'Assisi ${ }^{17}$, tralasciando quest'altro complesso esempio di santità francescana con un forte radicamento devozionale e culturale ${ }^{18}$.

Pur non sapendo attraverso quale forma orale o scritta fra Giuseppe derivò la metafora antoniana, fuoco, pentola e schiuma costituiscono elementi chiave dell'articolato linguaggio usato dal frate di Copertino, ma riferito da un teste durante il processo imbastito subito dopo la morte del carismatico religioso della Grottella. Forse "l'illitterato" fra Giuseppe l'avrà potuta derivare a seguito di una lettura personale dei testi del correligionario medievale, oppure dopo l'ascolto dei cicli di predicazione, o per altre pratiche che scandivano i tempi del convento. Diversamente dall'esempio antoniano, indirizzato ad un vasto pubblico, il linguaggio simbolico usato dal religioso del primo Seicento costituisce un ammonimento diretto ad una specifica categoria sociale: un confratello. A costui si rivolse il mistico frate al fine di orientarlo verso una pratica sacramentale che, proprio in epoca post-tridentina, si venne a modificare, per incidere sempre più profondamente sulle coscienze dei fedeli, tanto laici quanto ecclesiastici ${ }^{19}$. Affidata ad un corpo di ministri specializzati, morigerati, ben preparati e istruiti ${ }^{20}$, tale pratica doveva assumere, da un lato, la funzione di persuasione, di controllo, di orientamento dei comportamenti o condotte dei fedeli; dall'altro, doveva accentuare quella dimensione intimista, indagando le colpe che toccavano la sfera più privata dell'uomo, la sessualità ${ }^{21}$.

\section{i. Cibo, cottura, gesti: un codice figurato}

La metafora josefina della pentola sul fuoco ha generato un certo fascino tra gli studiosi da quando nel 2003 si è cominciato a studiare con rinnovati

17 Novi Chavarria 2004: 215-231, 227; Sodano 2004: 233-251, 244-247; Gentilcore 2004: 273-296, 282; Nestola 2004: 27-42, 32-34, 39; Spedicato 2009a: 495-512, 507-508; Fiorelli 2013: 41-50, 42.

18 Sallmann 1994: 77-78; Spedicato 2009b, 17-31.

19 Molto ricca è la bibliografia su questo tema che nell'ultimo ventennio ha attirato sempre più l'attenzione storiografica: Rusconi, 2002; Brambilla 2000; Romeo 1997; Prosperi 1996. Proiettato sullo spazio portoghese: Marques 2000: 445-459. Sul sistema di vigilanza dei comportamenti, credenze religiose e dottrinali per l'epoca moderna: Paiva 2011: 261-309; Idem 2013: 259-302.

${ }^{20}$ Mancino 2000. Per il territorio portoghese: Fernandes 2001: 426-438.

21 Flandrin 1981. Studi specifici sul Portogallo: Carvalho 2011: 96-129; Gouveia 2015. 
strumenti analitici e metodologie storiografiche la figura di fra Giuseppe $\mathrm{Desa}^{22}$. Non sempre, tuttavia, è stata data la dovuta considerazione a questo aneddoto, e molto generiche o fuorvianti sono state le argomentazioni suscitare da questa densa immagine culinaria, che costituisce un episodio di vita quotidiana apparentemente povero di significato. Alle prime e deboli proposte avanzate da Marcella Campanelli e da Mario Spedicato rispettivamente nel 2003 e nel 2009, hanno fatto seguito ipotesi interpretative più audaci, come quelle suggerite nel mio contributo del 2004 e ancora in quello del 2009, nel tentativo di individuare gli attori principali del sintomatico spazio in cui era ambientato il metaforico linguaggio josefino. Soprattutto in tale ultimo percorso, difatti, oltre a considerare una sorta di intercambiabilità di genere tra gli attori/destinatari di quella metafora, veniva identificata pure una precisa comunità a cui faceva riferimento il frate autoctono, attraverso coinvolgenti immagini tratte dall'armamentario medievale. Dopo diversi lustri dedicati al francescano pugliese, a seguito della comparazione con altri modelli agiografici e di applicazione territoriale dei dettami tridentini, nonché seguendo i molteplici approcci metodologici sulle pratiche alimentari e suoi articolati contesti socio-culturali, la sensazione è che oramai ci siano maggiori elementi per decifrare il codice usato dal mistico religioso di epoca moderna.

D'accordo con le proposte dello storico dell'alimentazione Massimo Montanari o degli antropologi Claude Lévi-Strauss e Mary Douglas, o ancora del semiologo Roland Barthes secondo cui l'alimentazione è una pratica che sostituisce, riassume o segnala altri comportamenti ${ }^{23}$, la proposta intende destrutturare il linguaggio metaforico usato dal religioso italiano, cercando di individuare non solo il legame tra arte culinaria e dimensione simbolico-culturale ricollegabile al sacramento della confessione, ma pure di rintracciare i modelli ideologico-culturali ai quali il frate francescano attingeva.

Fra Giuseppe da Copertino, d'accordo con le fonti agiografiche, leggeva e scriveva stentatamente ${ }^{24}$, non aveva la preparazione culturale e teologico-biblica del dotto confratello Antonio di Lisbona. Costui, oltre che nella città natale sulle rive del Tejo, si era formato anche in altri importanti centri religiosi come Coimbra, ricchi di patrimoni librari e di religiosi che avevano circolato in altri ambienti

22 Campanelli 2004: 267-268; Spedicato 2009: 505; Nestola 2004: 41-42; Eadem 2010: 19-64, 38-40.

${ }_{23}$ Montanari 2006; Lévi-Strauss 1966; Idem 2006: 425-448; Douglas 1972: 61-81; Barthes 1961: 977-986.

${ }^{24}$ Basato sul quadro culturale del clero di Terra d'Otranto: Spedicato 2009a: 503-504. Per l'epoca moderna la situazione culturale del clero portoghese è simile a quella italiana settentrionale e meridionale, e rimangono molte lacune circa la conoscenza della cultura letteraria del clero parrocchiale e regolare lusitano: Campanelli, 2003: 42-44; Sangalli, 2007: 108-111; Palomo 2006: 41-42, 49-50. Altrettanto carente era la preparazione del clero parrocchiale di una periferica diocesi del Portogallo settentrionale: Rocha Nunes 2014: 59-78. 
«Et il fuoco fa che la pignata mandi sopra la schiuma»: quando la lussuria sta in cucina?

Santi francescani italo-lusitani e disciplina sociale tra medioevo e prima etá moderna

culturali europei ${ }^{25}$. Ciononostante, $i$ frate italiano ha saputo incarnare tanto valori dell'ordine di appartenenza come obbedienza, umiltà e castità, quanto rinnovare il carisma di figure centrali della santità di epoca medievale come il serafico patriarca S. Francesco, o come il grande predicatore e taumaturgo portoghese. D'altra parte di tali santi mistici e contemplativi riconosciuti universalmente, il conventuale pugliese era particolare devoto, nonché attivo promotore presso altri fedeli.

Il nuovo percorso analitico proposto nelle prossime pagine si concentra su quello che si può definire il rituale del 'cuoco-matematico', ossia la peculiare tecnica di cottura suggerita dal contemplativo religioso pugliese, il quale era solito servirsi di un linguaggio metaforico particolarmente efficace per i suoi contemporanei. Come cercheremo di dimostrare, usando alcuni topici che riprendono la similitudine antoniana della pentola posta sul fuoco, in maniera altrettanto cauta fra Giuseppe suggeriva il giusto atteggiamento per prepararsi ad una buona confessione. Centrandosi su una particolare tecnica di cottura, su un sintomatico cibo, su una sequenza precisa di atti e di gesti, il frate alludeva ad un vero e proprio processo di purificazione ed eliminazione dei vizi dell'anima oltre che del corpo. A tale salutare pratica si poteva accedere dopo aver attraversato un preciso percorso psicologico, il quale necessitava di una calcolata arte culinaria affinché il corpo traesse pieno nutrimento spirituale.

\section{L'ARITMETICA MORALE DEL CUOCO-MATEMATICO}

Questa premessa permette di inquadrare meglio il valore simbolico della metafora culinaria accennata in esergo, e che presenta diversi elementi riconducibili alle similitudini dei Sermones antoniani. L'episodio agiografico si può ricostruire prevalentemente attraverso le fonti processuali, in particolare attraverso la deposizione di uno dei testimoni escussi nella diocesi di Nardò ${ }^{26}$. In questa circoscrizione ecclesiastica di Terra d'Otranto ricadeva la terra di origine del frate, che visse in questo piccolo centro agricolo prima di essere spostato nel 1638 in altri centri francescani, dietro ordine del Sant'Uffizio di Roma. Dalla deposizione del testimone, un confratello originario della stessa provincia napoletana, non è possibile tuttavia stabilire se sia un momento della vita del copertinese relativo agli anni trascorsi in Puglia, prima che venisse trasferito in altri conventi del centro-nord Italia; oppure ebbe come sfondo centri francescani come quello di Assisi, dove i due ebbero modo di incontrarsi ${ }^{27}$.

Il fatto che questo aneddoto agiografico non venga riportato dalle biografie ufficiali costituisce una assenza sintomatica, da inserire, verosimilmente, nella com-

25 Caeiro 1982:145-169; Idem 1981: 136-164; Cruz Coelho 1996: vol. I, 179-205; Blanco 2002: $127-147$.

${ }^{26}$ Campanelli 2004: 253-271.

27 Processo 2003: 246-257. 
plicata dinamica dell'iter di canonizzazione di quel frate considerato un "illetterato"28. Effettivamente fra Giuseppe, seppure attraverso un linguaggio metaforico, propose argomentazioni e concezioni teologico-morali che sarebbero dovute passare al vaglio di diversi teologi o canonisti: sia gli esperti delle Congregazioni che avrebbero potuto rallentare o addirittura compromettere l'iter di riconoscimento della santità del religioso che in vita era stato esaminato dal tribunale dell'Inquisizione; sia i censori preposti alla revisione delle biografie redatte dopo la sua morte.

In questo senso bisogna inquadrare le caute reticenze usate dal biografo Domenico Bernini, nell'opera Vita del venerabile padre fr. Giuseppe da Copertino de' minori conventuali, pubblicata nel 1723, e costruita meticolosamente sui processi romani, dopo l'elaborazione di altri esempi bio-agiografici ${ }^{29}$. L'erudito e accorto autore romano preferì escludere l'aneddoto dialogico della metafora culinaria, concentrandosi piuttosto su altre espressioni ed affermazioni riferite dal frate copertinese. Un intero capitolo difatti è dedicato a quei detti josefini, estratti dai processi e presentati al pubblico di lettori tra cui il dedicatario papa Innocenzio XIII, come pedagogico ed edificante esempio ${ }^{30}$.

Anche per la sintomatica autocensura berniniana e per l'esclusione dalle fonti josefine a vasta diffusione, la deposizione rilasciata durante il processo neretino è meritevole di essere seguita dalle dirette parole del testimone oculare, fra Giuseppe da Carmiano. Costui, incaricato di redigere una biografia dell'omonimo confratello frequentato in diversi contesti conventuali, durante il processo del 1664 così depose sul filo della memoria:

Un'altra fiata, fra Giuseppe mi disse che mi voleva far cuoco, e mi disse che il buon cuoco, per cucinare bene la minestra e la carne, prima la lava, e lava anco la pignata, e dopo vi mette l'acqua e l'accosta al fuoco; et il fuoco fa che la pignata mandi sopra la schiuma, la quale si deve levare con la cucchiara, e poi per virtù dello stesso fuoco la pignata incomincia a bullire, et il cuoco deve per questo più presto tirare a dietro la pignata, che metterla vicino al fuoco, perché vicino al fuoco si manda fuori il grasso. E poi disse che la pignata era l'anima nostra, e che la dovevamo pulire bene, e quella schiuma è la raccordanza delle colpe, che devesi levare con la cocchiara del pentimento; poi incomincia a bullire, cioè a ricordarsi dell'amore di Dio, ma non per questo dovemo presumere e confidarsi e dire che già semo santi, ma per humiltà dovemo tornare a dietro e dire che non semo degni di tanta gratia e che siamo peccatori, perché estremamente il grasso della gratia di Dio si rovescia fuora ${ }^{31}$.

\footnotetext{
${ }^{28}$ Gotor 2004: 101-102.

29 Rotondó 1967: 364-365; Mormando 2011.

30 Bernini 1722: 482-503; Nestola 2015: 334-335.

31 Processo 2003: 253. Nel testo il grassetto è mio.
} 
Nonostante sia una testimonianza processuale trascritta da un notaio, la dettagliata ammonizione josefina manifesta un valore fortemente simbolico, evidente fin da alcuni aspetti fonetici della metafora culinaria. Per esempio, gioca sulla ripetuta assonanza delle parole «cuoco» «fuoco», quasi a voler riprodurre onomatopeicamente l'ambiente delle cucine, e soprattutto il suono dell'ebollizione della pentola esposta alla fonte di calore. Ancora, contrappone l'azione purificatrice dell'acqua a quella del fuoco applicata tanto alla carne quanto alla pentola, ossia all'ingrediente naturale e all'utensile culturale che rappresentavano rispettivamente il corpo e l'anima, secondo quanto spiegò lo stesso religioso. Effettivamente anche la tecnica descrittiva, la scelta dell'attore principale e del luogo dove si svolgeva la scena, degli strumenti utilizzati o delle modalità di cottura sono altrettanto sintomatici di un messaggio che cercheremo di destrutturare.

Tra i diversi spazi claustrali, la cucina non rappresentava un ambiente neutro. Piuttosto, costituisce il luogo dove avviene la trasformazione del cibo che passa dallo stadio crudo al cotto, dal solido al fluido, dal liquido al semi-liquido, dal naturale al culturale ${ }^{32}$. Una sorta di cucina sacralizzata quella presentata da fra Giuseppe al confratello, per il quale anche la carne e la minestra, ossia l'alimento solido e quello semi-liquido, vengono trasformati in salutare alimento spirituale. Non a caso quell'avvertimento dal valore altamente morale è incentrato su un cibo simbolico, identificativo di un preciso status. La carne costituiva un alimento di lusso ancora per gran parte delle tavole del primo Seicento ${ }^{33}$, e fin dall'epoca medievale negli ambienti religiosi rappresentava una sorta di metonimia del peccato: un cibo pericoloso in quanto destinato ad alimentare la lussuria ${ }^{34}$. Rifiutata come fonte di nutrimento dal frate copertinese, che addirittura la rigettava dopo averla assunta per obbedire agli ordini dei superiori, pietanze a base di carne costituivano portate frequenti nella dieta dei conventi francescani pugliesi di epoca moderna. Ricette conventuali preparate secondo modi e tempi di cottura diversi come arrosti, bolliti, stufati etc. ${ }^{35}$. Torneremo più avanti sulla specifica modalità di preparazione alla quale si riferisce fra Giuseppe nell'istruzione data al suo giovane interlocutore.

É necessario approfondire lo status del destinatario della metafora culinaria josefina, la quale fin dalle prime esortazioni riproduce un percorso didattico indirizzato ad una precisa categoria sociale: un religioso. Fra Giuseppe da Carmiano era sì un individuo che aveva fatto voto di castità per entrare nella regola francescana, ma si stava preparando pure per distinguersi per cultura

\footnotetext{
32 Montanari 2004, particolarmente il capitolo II L'invenzione della cucina; Sarti 2003: 211-225.

33 Drumond Braga 2015: 35-85.

${ }^{34}$ Casagrande, Vecchio 2000: 147-180.

35 D'Ambrosio, Spedicato 1998: 55-56.
} 
e dottrina rispetto agli altri. Non abbiamo molti elementi per ricostruire la biografia di questo testimone che depose nell'interrogatorio del primo processo ordinario sulla vita, virtù e miracoli dell'omonimo frate di Copertino ${ }^{36}$, riferendo pure diversi episodi appresi de auditu o de visu, e avvenuti in centri francescani dove il copertinese aveva soggiornato, quali il piccolo convento della terra natale, o quelli più importanti delle città di Lecce, di Monopoli e di Assisi ${ }^{37}$. Tra le prime risposte date all'interrogatorio, il teste dichiarò di avere quarantacinque anni ${ }^{38}$, di essere figlio di Pietro Antonio Provenzale e di Giovanna Manzo, di essere maestro in teologia, qualifica questa che confermò anche al momento della firma della propria testimonianza, a cui aggiunse pure di aver ricoperto la carica di ministro della provincia di S. Nicolò. Maestro di teologia, provinciale, ma anche predicatore, d'accordo con quanto dichiarò in una delle risposte del lungo interrogatorio: "Quando viddi fra Giuseppe in Assisi lo conobbi per haver io andato là per vedere il corpo (come si dice) del nostro padre S. Francesco, perché là vi predicai un Advento" ${ }^{39}$. Anche per tali elevati gradi culturali dichiarati si può intuire che, con quella ammonizione moraleggiante, il frate di Copertino volesse orientare quel giovane studioso di teologia, guidando sia il momento della confessione personale, sia le sue capacità mnemotecniche. Queste erano modalità dell'organizzazione del pensiero e della memoria da utilizzare tanto durante il rito sacramentale nel ruolo di penitente, ossia nell'esercizio della propria confessione; quanto in quella di confessore di altri fedeli. Le capacità di rafforzamento della memoria, ancora, facevano parte anche dell'ars oratoria ad uso dei predicatori, i quali le avevano derivate dal mondo classico per meglio organizzare il discorso fissando nella mente alcune componenti essenziali come le imagines agentes ${ }^{40}$.

Nel caso della metafora josefina, proprio in qualità di "buon cuoco" al religioso venne illustrata dettagliatamente quella ricetta culinaria che, organizzata come uno schema visivo, prevedeva precisi gesti, rapide successioni e passaggi della pentola, al fine di ottenere un succulento bollito di carne. D'altra parte fu lo stesso teste fra Giuseppe Provenzano a riferire ordinatamente di alcuni episodi appresi attraverso dei manoscritti biografici sul confratello. Episodi avvenuti nelle cucine conventuali, durante il noviziato di fra Giuseppe da Copertino, quando questi "faceva li più vili esercitij del

${ }^{36}$ Un sintetico profilo di questo teste, chiamato a deporre nel processo periferico del 1664 celebrato a Nardò: Campanelli 2004: 259.

37 Campanelli 2013: 17-27.

38 Processo 2003: 246-257.

39 Ibidem, 246.

40 Sui moduli mnemonici basati su luoghi, ordine, immagini: Bolzoni 1995: XVIII. 
«Et il fuoco fa che la pignata mandi sopra la schiuma»: quando la lussuria sta in cucina?

Santi francescani italo-lusitani e disciplina sociale tra medioevo e prima etá moderna

convento, scopando, lavando piatti” ${ }^{41}$. Ancora fu lo stesso teologo a deporre circa il volo di fra Giuseppe fino all'altare di Sant'Antonio da Padova, verificatosi a Monopoli alla sua presenza e di altri frati ${ }^{42}$. Anche questi ultimi episodi vennero trattati con molta cautela dal puntuale Bernini, pratico nel trattamento delle fonti processuali romane.

Ciononostante è evidente come la prossimità tra i due omonimi confratelli fosse molto forte oltre che di antica data, secondo quanto si deduce anche dalla seguente preliminare dichiarazione: "Quando lo vidi [fra Giuseppe da Copertino] nella Grottella, fu la causa che essendo io novitio, mi condusse là il padre maestro de novitij a vedere il padre fra Giuseppe, il quale mi fé una lettione di moralità, essendo presenti molti frati" ${ }^{43}$.

Possiamo ipotizzare che il Provenzano si riferisse proprio alla indelebile metafora culinaria del carismatico frate, riferita con quel ricco ed indimenticabile linguaggio simbolico? Non possiamo rispondere esattamente a questa domanda; tuttavia, delineato il profilo del destinatario dell'articolato messaggio josefino, cerchiamo di rilevare alcune differenze rispetto alla pentola in ebollizione a cui allude in forma efficace ma generica Sant'Antonio nei suoi Sermones. Difatti, mentre la simbologia del teologo medievale si concentrava sull'utensile posto sul fuoco dirigendosi ad un pubblico diversificato che non sempre poteva contare su una dieta a base di proteine animali, o che difficilmente conosceva l'esatto procedimento per la buona preparazione del bollito di carne; nell'esempio josefino il linguaggio metaforico diretto ad un preciso interlocutore si focalizzava proprio sul soggetto, sugli ingredienti, sugli utensili e sulle tecniche di una calcolata arte culinaria. Una professione questa che richiedeva doti di competenza, di abilità e di precisione, analogamente ad altre specializzazioni come quella del medico o del confessore, i cui requisiti di esperienza e di maturità erano necessari per salvaguardare tanto la salute del corpo quanto quella dello spirito ${ }^{44}$. Per un religioso pratico delle cucine conventuali come era fra Giuseppe, la metafora del cuoco poteva essere certamente frutto dell'esperienza personale, ma forse era qualcosa di più.

Recentemente Raffaella Sarti, focalizzandosi su diversi territori europei, ha evidenziato il ruolo privilegiato occupato soprattutto dagli uomini nella preparazione degli alimenti, rispetto alle donne ${ }^{45}$. Non soltanto questioni di natura materiale, anche quelle simboliche contribuivano a privilegiare i primi, considerati più affidabili rispetto alle seconde ${ }^{46}$. D'altra parte, seppure riguardante un altro contesto territoriale, in quegli stessi anni il dramma-

\footnotetext{
41 Processo 2003: 248.

${ }^{42}$ Ibidem, 251.

43 Ibidem, 246.

${ }^{44}$ Turrini: 1991.

45 Sarti 2003: 211-225; si veda anche la differenza tra cuciniera e cuoco: Montanari 1991: 209.

46 Sarti 2012: 87-158.
} 
turgo inglese Ben Jonson (1572-1637) nella commedia The Staple of News (1625), definiva il cuoco "un architetto, un ingegnere, un soldato, un filosofo e scienziato, e in ogni matematica è versato" ${ }^{47}$. Una testimonianza preziosa questa, un utile indizio per decifrare meglio le molteplici competenze a cui, forse, voleva alludere il frate, abituato soprattutto alle cucine conventuali. Verosimilmente l'ammonizione josefina intendeva indirizzare il giovane confratello a diventare un "buon cuoco" e - d'accordo con la commedia inglese - "versato in ogni matematica", perito pertanto nel conteggio di quantità astratte. Verosimilmente si trattava del conteggio non soltanto della quantità degli alimenti e delle loro combinazioni, quanto dei loro tempi di cottura. Una sorta di "aritmetica morale", per riprendere la felice formula usata da John Bossy nel 1988, ripresa poi in uno studio del $1998^{48}$.

Nella veste di cuoco-matematico quel religioso avrebbe dovuto avere una precisa conoscenza dell'ordine dei peccati, della loro classificazione per genere, specie e gravità, delle dinamiche penitenziali connesse. Una organizzazione che aveva una lunga storia, basata su specifiche configurazioni dei vizi capitali come il settenario, che Gregorio Magno aveva fissato fin dall'epoca alto medievale ${ }^{49}$, e a cui lo stesso Antonio da Lisbona si riferiva nei suoi sermoni. Lordine tassonomico gregoriano, iniziando con la superbia e terminando con la lussuria, poteva coesistere, tuttavia, con un'altra organizzazione, la quale prevedeva una scansione ternaria dei peccati, divisi tra peccati di pensiero, di parola e di azione, secondo le formule di San Gerolamo e di Sant'Agostino ${ }^{50}$. Alla gerarchia verticale dei peccati si associava, pertanto, un percorso psicologico che, sviluppando un andamento interiorità/ esteriorità, stabiliva un preciso ordine nel sistema dei vizi e nell'universo della colpa. Tale concezione dinamica del peccato, proposta attraverso lo schema ternario, permetteva di utilizzare la formula agostiniana anche in relazione alla pratica penitenziale, di modo che ai peccati di pensiero avrebbe dovuto corrispondere la compunzione del cuore; a quelli di parola la confessione della bocca; terminando con i peccati di azione, a cui la soddisfazione delle opere avrebbe dovuto dare esito. D'accordo con Bossy, inoltre, dopo il XVI secolo venne predicato un sistema morale basato sui Dieci comandamenti; e soprattutto a seguito della diffusione del catechismo il Decalogo divenne il protagonista del sistema morale ${ }^{51}$.

\footnotetext{
${ }^{47}$ Si riprende la commedia del poeta e drammaturgo inglese riportata in traduzione italiana: Montanari 1991: 193-194.

48 Bossy 1998: 87-116.

49 Casagrande,Vecchio 2000: 181-224; Barata 2014: 81-83.

${ }^{50}$ Casagrande,Vecchio 2000: 181.

51 Bossy 1998: 89; Fernandes 1995: 47-65.
} 
A ben guardare anche la metafora culinaria riferita da fra Giuseppe presenta un rigoroso codice aritmetico, al di là dei giochi fonetici e delle contrapposizioni corpo/spirito, liquido/solido, crudo/cotto, puro/impuro, naturale/culturale degli alimenti o degli utensili culinari adoperati. In pratica la successione morfologica della ricetta organizzava il discorso su un sistema numerico gravitante intorno alle azioni eseguite dal buon cuoco-matematico. Era proprio costui che conosceva quelle simboliche successioni e concatenate combinazioni. Ad esempio, da esperto professionista eseguiva sette azioni secondo un preciso ordine come: lavare la carne; lavare la pignata; mettere l'acqua in quel recipiente; accostarlo al fuoco; levare la schiuma che si è formata; allontanare la pentola dal fuoco; non avvicinare quel recipiente alla fonte di calore. Con questi inviti a fare e a non fare, a mettere e a togliere, ad essere attento durante il processo di cottura, sembra tuttavia che venga suggerita pure una organizzazione basata sulle prescrizioni del Decalogo, i cui precetti guidavano la morale e l'imperativo comando o l'esplicito divieto di quanto si doveva fare e quanto si doveva evitare.

Nel caso josefino, proprio da cuoco pratico il suo interlocutore, tanto nella veste di penitente quanto in quella di confessore, avrebbe dovuto conoscere le reazioni attivate dal fuoco, i cui effetti agivano sia sulla pentola, sia sul suo complesso contenuto di acqua, di carne, di schiuma. Vigile durante il processo della preparazione del cibo, il professionista del fuoco conosceva le dinamiche interne/esterne attivate dalla fonte di calore: questa difatti, oltre a determinare la cottura dell'alimento, favoriva la risalita delle impurità e l'ebollizione del liquido dalla pentola, fino a causare la fuoriuscita del grasso a seguito di una prolungata ed eccessiva cottura.

D'altra parte, il "buon cuoco" al quale si riferiva fra Giuseppe costituiva una categoria specifica: un religioso che, oltre alla confessione personale, poteva assolvere e cumulare il ruolo di confessore di altri fedeli. Proprio per questa caratteristica, l'agente del sacramento della confessione era specializzato nella "aritmetica morale", nella conoscenza ed applicazione delle operazioni matematiche tra numeri carichi di valori simbolici come il sette, il tre, il dieci. Numeri tenuti a mente ed applicati nel calcolo morale. Tali cifre e combinazioni numeriche riguardanti vizi del corpo o dell'anima erano riportate non solo nei testi sacri ma anche nei trattati di morale e nei manuali per confessori; elencate da predicatori dall'alto dei pulpiti; trattate nei testi legislativi sinodali ${ }^{52}$ e, non da ultimo, raffigurate con molta cautela dai pittori in quelle opere che insistevano su gesti espressivi come il computo digitale ${ }^{53}$.

52 Miele, 1997: 15-64; Romeo, 1997; Mancino, 2000; per l'area portoghese: Paiva, 2000: 9-15; Idem, 2009.

${ }_{53}$ Schmitt 1984: 1-28; Bertelli-Centanni 1995; Dalli Regoli 2000; Frugoni 2010: 102-103. 
A titolo di esempio ed in mancanza di studi approfonditi anche sul sistema idoneativo per l'amministrazione della penitenza o sulle forme di reclutamento dei confessori di questo territorio del Mezzogiorno italiano, si veda quanto promulgato negli stessi anni della seconda metà del Seicento dal vescovo di Lecce, Luigi Pappacoda. Tale sede diocesana era adiacente a quella dove risiedevano i due protagonisti di quell'indimenticabile testimonianza josefina. In questa preminente circoscrizione della provincia di Terra d'Otranto, dove si erano verificati pure alcuni casi di sollecitatio ad turpia segnalati al tribunale dell'Inquisizione di Roma negli anni 1626-28 ${ }^{54}$, il presule Pappacoda legiferava secondo un catalogo numerico nel sinodo stampato in latino del maggio del 1669 , ma convocato nel $1663^{55}$. Con una lunga esperienza pastorale, il vescovo aveva una formazione in utriusque iuris piuttosto che in teologia. Del lungo elenco di trenta avvertimenti che componevano l'Instrutione per li Confessori, soprattutto il punto quattordici presentava una articolata organizzazione mnemonico-morale alla quale attenersi durante la pratica del sacramento di diverse categorie sociali ${ }^{56}$ :

Venendo alla confessione delli peccati, esorterà il penitente a dire
tutti quelli de'quali ricorda \& havendo bisogno d'esser interrogato,
l'interrogarà secondo che giudicarà conveniente allo stato di quello, o
dei comandamenti del Decalogo, o de' sette peccati capitali, o de i cin-
que sentimenti, o delli precetti della Chiesa, o dell'Opere della Miseri-
cordia, e particolarmente dell'esercitio proprio del medesimo penitente,
come delli peccati che ordinariamente si sogliono commettere nella
mercantia, nell'artificio, e nella militia, nell'Avocatione, nella procura,
nel giudicare, nel notariato, nel magistrato e simili, interrogando ciascu-
no, secondo la sua professione ${ }^{57}$.

Redatta in italiano, probabilmente per facilitare la diffusione di quelle raccomandazioni, in quella lista numerica sinodale ad uso dei ministri ritenuti idonei, accanto a tipologie professionali - interrogate secondo specifiche tassonomie - non si tralasciavano altre componenti della comunità come donne, "figliuoli o altri penitenti semplici" ai quali era dedicato il paragrafo quindici ${ }^{58}$. Per tutti, ancora, erano perentorie le istruzioni dei successivi punti, dove si proponevano altri quesiti intorno ai quali avrebbe dovuto gravitare il confessore con caute domande. Secondo quanto esemplifica la seguente

${ }_{54}$ Biblioteca Apostolica Vaticana, Barb. Lat. 6334, fols. 35 r, 207 v-208 r; Ivi, 6335, fols. 71 r, 75 r, 359 v; Ibidem, 6336, fol.120 r.

55 Secunda Synodus Diocesana ab Aloysio Pappacoda Episcopo Liciensi celebrata, Romae, Success. Mascardi, MDCLXIX. Su questo sinodo e la cultura del tempo: Pisanó 1995: 95-137.

56 Secunda Synodus Diocesana 1669: 97-104.

57 Ibidem, 100.

58 Ibidem. 
«Et il fuoco fa che la pignata mandi sopra la schiuma»: quando la lussuria sta in cucina?

Santi francescani italo-lusitani e disciplina sociale tra medioevo e prima etá moderna

disposizione, inoltre, quelle domande si ricollegavano ad una sequenza numerica costituita da sette avverbi latini, facilmente memorizzabili:

Non lasciarà mai di domandar il numero delli peccati e se il penitente non saprà dirlo precisamente gli domandará che dica quel numero che pensa essere più appresso alla verità, dico più o meno, o vero, essendo vissuto in stato di peccato mortale, dichiari per quanto tempo.

Dimandarà delle circostanze, che de' veniali fanno mortali, e quelli che mutano la specie comprese in quelle sette del verso quis, quid, ubi, quibus auxilijs, cur, quomodo, quando ${ }^{59}$.

Insomma anche da questa indicazione sinodale, che riprendeva un verso delle opere teologiche di san Tommaso d'Aquino, è evidente come il momento della confessione costituisse un dialogo tra penitente e confessore. Un colloquio meticoloso, basato su formule linguistico-numeriche facilmente memorizzabili ma che spingevano il penitentente/confessore a contare quantità astratte, fornendo sia l'esatto numero dei peccati commessi, sia il conteggio del tempo di quella condizione veniale o mortale. Quello proposto dal presule di Lecce costituiva uno schema mnemonico costituito da formule linguistico-numeriche, che presentava non poche cautele anche nella comunicazione non verbale, ossia nella mimica e nella esecuzione di atti e gesti durante la celebrazione del sacramento. Seguendo tale regola, lontano dagli occhi di altri fedeli, si doveva stabilire un proficuo dialogo tra i due esclusivi protagonisti del rito penitenziale, d'accordo con il seguente punto:

si guardará di non far atto, mentre confessa, né gesto alcuno, per lo quale qualche circostante possa accorgersi della gravezza del peccato, \& anco per non spaventare il penitente, acciò non tacesse altri simili e maggiori peccati, anzi li faccia animo, ne lo riprenda se non dopo che havrá compito d'interrogarlo, allora li faccia conoscere la bruttezza de' suoi peccati, e particolarmente de i più enormi, e de i più frequenti, forzandosi d'indurlo al dolore d'essi \& al fermo proposito di mai più commetterli con proponerli l'offesa ch'ha fatta a Dio, tanto suo Benefattore, il quale l'ha aspettato fin hora a penitenza, e che egli s'è servito de' doni di Dio, cioè della sanità, della ricchezza, della vita in offesa dell'istesso Dio, li proponerá anco la pena preparatali nell'inferno, \& il bene del Paradiso che há perduto e la gravezza della penitenza, che ordinariamente impongono li Sacri Canoni, li quali per ogni peccato mortale sogliono dare sette anni di penitenza, ne saria senza molto frutto l'haver preparato qualche bello esempio al proposito da poterli addurre ${ }^{60}$.

\footnotetext{
${ }^{59}$ Ibidem, 100-101.

${ }^{60}$ Ibidem.
} 
Non é possibile dilungarsi ulteriormente su questo elenco sinodale e sue puntuali disposizioni che rimandano a precise concezioni del peccato e dinamiche escatologiche ${ }^{61}$. Ciononostante, emergono alcune conferme rispetto a quanto formulato dal carismatico esponente dell'ordine francescano fra Giuseppe da Copertino.

In quella articolata metafora culinaria, difatti, il frate riproponeva un facile schema mnemonico, utile per visualizzare gli elementi astratti di una serie. Una serie che andava dal cinque, al sette, al dieci, e difficile da ricordare tanto a voce, quanto senza l'aiuto del computo digitale al fine di non attirare la curiosità di altri fedeli. Durante la confessione, la voce di certo aveva il potere di informazione e di scambio dialogico, codificato nell'articolazione del linguaggio parlato e scritto. Al linguaggio orale mancava però l'intervento diretto di un contesto immediatamente visibile, cioè l'azione, ma il codice figurato josefino contribuiva a superare tale difficoltà mnemonica, velandola al contempo con passaggi teologici prudenti, inculcati attraverso un linguaggio fatto di esemplificazioni tratte dal mondo quotidiano, in particolare dall'universo delle cucine conventuali.

Nel tentativo di riprodurre esemplificazioni di gesti che non fossero troppo espliciti, forse in questo senso possiamo intendere le cautele dei pittori, i quali dovevano centrarsi proprio sulle mani per tradurre una precisa tassonomia morale. Una schematizzazione resa dalla parola minuziosa e più rigida rispetto all'elaborazione visuale, che offriva margini di interpretazione più ampi ed elastici, soprattutto per un pubblico esterno al sacramento e alle sue dinamiche penitenziali. Anche per questo, ambientare i santi in contesti diversi da quelli popolati dagli uomini, poteva costituire un espediente efficace per articolare un cauto discorso gestuale, riconducibile a specifici processi teologico-morali.

Seppure riferibili al primo quarto del Cinquecento, sono diversi gli esempi in cui proprio il mistico Sant'Antonio veniva raffigurato in ambienti topologici naturali, ma assorto nell'atteggiamento del computo digitale. Esemplificative sono due opere realizzate dal pittore portoghese Vasco Fernandes, come il trittico oggi al Museo di Arte Antica di Lisbona, o la pala proveniente da una chiesa di Santiago de Besteiros, località prossima a Viseu ${ }^{62}$. In tali potenti documenti iconografici ambientati tra paesaggi isolati, tra contesti tellurici e falesie rocciose, il prolifico pittore attribuisce un ruolo predominante a questo aspetto del teologo francescano, raffigurato nel mentre è concentrato su una specifica articolazione digitale di entrambe le mani. Potremmo dire "mani parlanti” quelle raffigurate dal Fernandes, le quali richiamavano i gesti tipici

\footnotetext{
${ }^{61}$ Delumeau 1978.

${ }^{62}$ Reis Santos 1945: 10; Rodrigues 1992: 144-151; Eadem 2007: 87-93.
} 
degli oratori dell'antichità d'accordo con Chiara Frugoni ${ }^{63}$, ma che finora sono stati ricollegati esclusivamente all'episodio agiografico della predica ai pesci, secondo quanto ha evidenziato di recente Dalila Rodrigues ${ }^{64}$. La rigorosa analisi della Rodrigues ha giustamente richiamato il ruolo della parenetica francescana soprattutto analizzando l'opera di Sant'Antonio che compone il trittico di Lisbona; così come ha inserito la tela raffigurante il santo portoghese della chiesa di Santiago de Besteiros (databile probabilmente al 1525) nel contesto del processo di rinnovamento voluto dall'erudito vescovo della diocesi di Viseu, D. Miguel da Silva ${ }^{65}$. Proprio considerando tali interessanti suggestioni si potrebbe aggiungere il fatto che questo attivo prelato fosse particolarmente attento alla presenza di predicatori autorizzati nel proprio territorio di giurisdizione, secondo le disposizioni sinodali promulgate nel 1527, d'accordo con José Pedro Paiva ${ }^{66}$.

Anche per la specificità di tale precoce iniziativa vescovile si dovrebbe rileggere il significato iconologico dell'opera del Fernandes costituita dal dipinto di Sant'Antonio, associato a quella di San Giovanni Battista (Fig. 1-2). Opere pittoriche che rappresentano in modo evidente il nesso sempre più forte stabilitosi tra predicazione e confessione del periodo liturgico quaresimale, in preparazione dell'adempimento del precetto ecclesiastico della confessione annuale e della comunione pasquale ${ }^{67}$. Tale interpretazione potrebbe trovare riscontro nel dipinto, dove il dotto santo portoghese è associato a San Giovanni che, non a caso, indigita un trionfante Agnus Dei. Dal canto loro le dieci dita del teologo francescano e le loro distinte parti sono raffigurate piegate o distese, chiuse o aperte, raggruppate o unite nella pressione dei polpastrelli, evidentemente fissate a seguire con il conteggio manuale un ordine logico gravitante su un preciso numero: il primo di una serie. Una tassonomia superba quella rappresentata con molta probabilità dall'artista, riconducibile tanto all'ordine morale del settenario quanto a quella del Decalogo, ed evidentemente segnalata nel gruppo manuale dell'indice della mano destra premuto sul pollice della sinistra. Con la predicazione antoniana si intendeva indurre i fedeli alla virtù della penitenza e all'osservanza delle prescrizioni del canone Omnis utriusque sexus, e di predicatori autorizzati e controllati dall'autorità vescovile diventavano i principali agenti di tale

63 Frugoni, 2010: 70.

${ }^{64}$ Rodrigues, Grão Vasco 2007: 87 e 92.

65 Ibidem, p. 93.

${ }^{66}$ Paiva, 2009: 8-9; Idem, 2013: 218-219. Su questo colto ed erudito prelato che governò la sede di Viseu dal 1526 al 1547, ebbe importanti incarichi nella penisola italiana, mantenne forti legami anche con figure di rilievo dell'episcopato italiano, ed ebbe alterne vicende con la corte portoghese: Idem 2006: 124, 187, 210.

${ }_{67}$ Rusconi 2002: 114-117. 
ministero pastorale di contro a quelli itineranti, reclutati tra il clero regolare, ma senza la necessaria licenza.

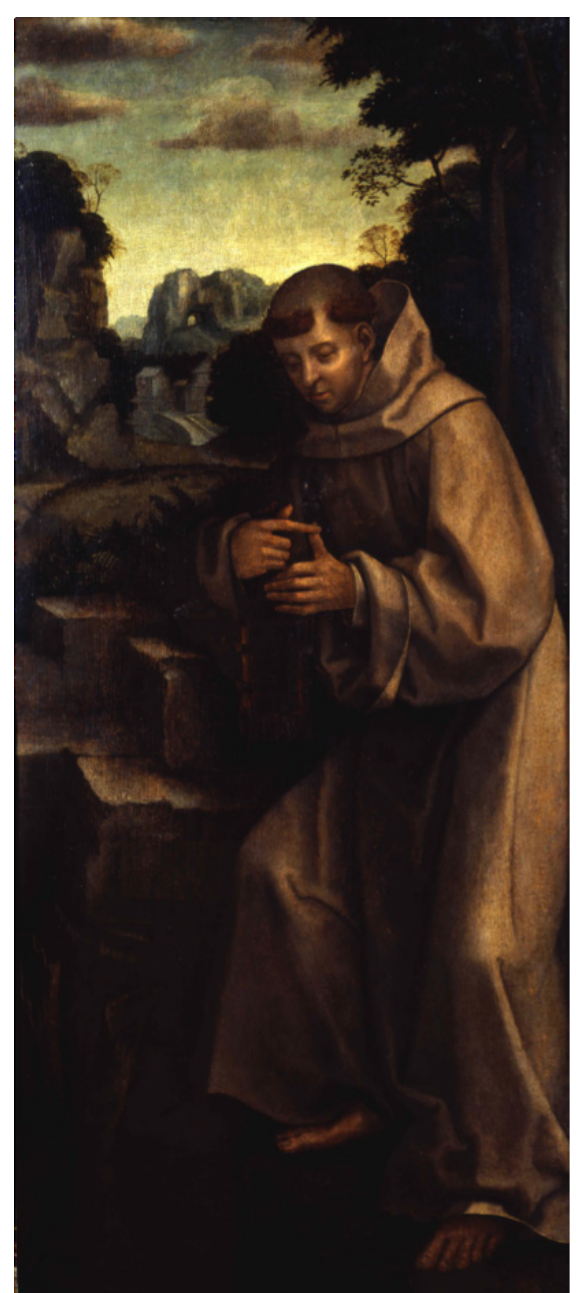

Figura 1: Vasco Fernandes, Sant'Antonio, 1525 c.

(Museu do Caramulo/Fundação Abel de Lacerda) 
«Et il fuoco fa che la pignata mandi sopra la schiuma»: quando la lussuria sta in cucina?

Santi francescani italo-lusitani e disciplina sociale tra medioevo e prima etá moderna

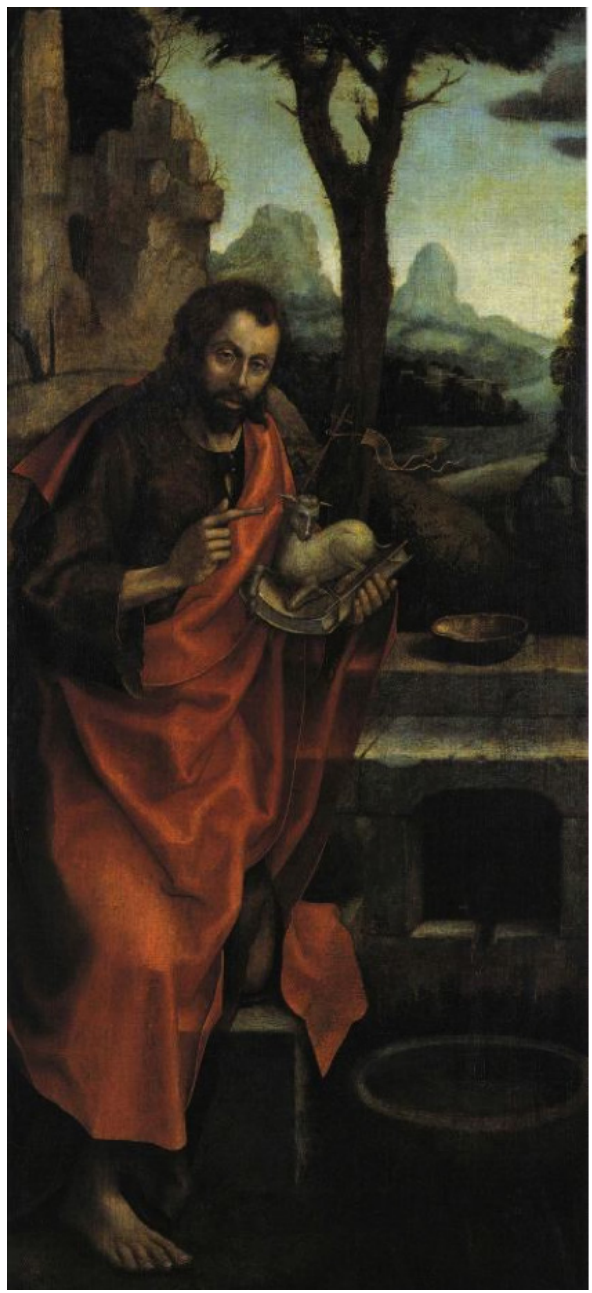

Figura 2: Vasco Fernandes, San Giovanni Battista, 1525 c.

(Chiesa di Santiago de Besteiros, Caramulo, Collezione privata)

D'altra parte Roberto Rusconi nel suo incisivo contributo ha messo in guardia sulla polimorfa rappresentazione della penitenza sacramentale e dell'ascolto della confessione dei peccati relativamente al periodo dalla seconda metà del XII e inizio del XVI secolo, evidenziando non pochi esempi del polimorfismo iconografico riscontrabile sia a seconda delle epoche e aree geografiche, sia dei supporti come libri, miniature, affreschi, pale ${ }^{68}$.

\footnotetext{
68 Ibidem, p. 161.
} 
Traducendo le immagini in parole, anche il codice linguistico-visuale josefino insisteva su precisi e calcolati gesti in cui mani e braccia erano protagonisti del rituale culinario: braccia in tensione, mani che afferrano, che impugnano, che eseguono azioni in uno spazio topologico definito ed in una lotta continua tra azione del fuoco, tempi di cottura, dinamiche della pentola e dei suoi contenuti, azione degli utensili necessari a non contaminare il cibo. Anche con quelle potenti imagines agentes l'illetterato fra Giuseppe, nel primo quarto del Seicento, metaforizzava ed enfatizzava quelle dettate dai sentimenti, dagli atteggiamenti o da altri interventi attivi dell'uomo. Nel ricco discorso metaforico josefino - un "bello esempio", per riprendere l'ammonizione sinodale del vescovo Pappacoda - è evidente lo sforzo di superare la dispersione nel tentativo di trovare un impianto unitario, riconducibile ad un insieme numerico dotato di una potente carica simbolica come l'organizzazione del settenario dei vizi o quella dettata dal Decalogo. Tale sistema poteva essere combattuto - o meglio controbilanciato - da un atteggiamento basato sulle virtù. In questo modo alla formula del $7+3$ si poteva aggiungere anche un sistema binario che privilegiava i comportamenti virtuosi. Una organizzazione di segno differente, che nella metafora josefina trova un chiaro riferimento nell'evocazione dell'umiltà contrapposta alla superbia della presunzione. Proprio tale sistema binario fa da sfondo nell'ultima parte della metafora, dove l'umiltà del peccatore viene contrapposta alla presunzione di chi si definisce santo e vuole ascendere alle gerarchie celesti, avvicinandosi a Dio.

\section{Conclusione}

Con questo evidente riferimento a quello che era considerato il primo dei peccati, pericoloso pure per i religiosi che si preparavano a seguire un iter di alta formazione dottrinale come nel caso dell'interlocutore josefino, si chiude il circolo vizioso avviato dalla lussuria, ultimo dei vizi carnali nonché il più intimo. Non a caso i suoi trasbordanti effetti erano paragonati a quelli di una pentola in ebollizione, come nell'immagine metaforica di ascendenza antoniana.

Attraverso i sapienti gesti del buon cuoco, esperto nei concatenati passaggi e nella misura dei tempi di cottura della carne, anche il grasso della grazia di Dio si manteneva come elemento nutritivo all'interno della pignata. Fra Giuseppe da Copertino si dirigeva al giovane confratello con un linguaggio povero di cultura teologica ma ricco di osservazione contemplativa, un procedimento figurato carico di simbologie teologiche che avrebbero potuto attirare le censure sulle opere degli agiografi, e per questo da loro tralasciato. Il mistico francescano proponeva una salutare ricetta che prevedeva una vigilata cottura della carne, di modo che era tenuto a freno pure il primo dei 
«Et il fuoco fa che la pignata mandi sopra la schiuma»: quando la lussuria sta in cucina?

Santi francescani italo-lusitani e disciplina sociale tra medioevo e prima etá moderna

vizi capitali, condannato tanto dal settenario quanto dal Decalogo. Il frate costruiva la sua formula mnemotecnica alla base dell'esame di coscienza attraverso un percorso che procedeva dall'anima al cuore, trasmettendo così un iter introspettivo paragonabile ad una regolata arte culinaria.

Eseguito dal buon cuoco-matematico, anche il bollito di carne a cui alludeva il frate pugliese rappresentava una modalità di cottura basata sulla purificazione dell'anima, attraverso una dinamica centrata sull'aritmetica dei peccati di pensiero, di parola e di azione, sulla loro meticolosa classificazione. Desideri, omissioni o atti, nei quali si sarebbero potuti consumare i vizi dell'anima come la lussuria, e altri peccati di carne o di spirito.

\section{RifERIMENTI}

\section{Fonti}

Roma, Biblioteca Apostolica Vaticana:

Barb. lat., 6334, lettere della Congregazione dell'Inquisizione per l'anno 1626.

Barb. lat., 6335, lettere della Congregazione dell'Inquisizione per l'anno 1627.

Barb. lat., 6336, lettere della Congregazione dell'Inquisizione per l'anno 1628.

Bernini, D. (1722), Vita del venerabile padre fr. Giuseppe da Copertino de' minori conventuali, Ludovico Tinassi e Girolamo Mainardi, Roma.

Processo per la beatificazione de la canonizzazione del servo di Dio fra Giuseppe Desa di Copertino (2003), O. Mazzotta, M. Spedicato (a cura di), Edipan, Galatina.

S. Antonii Patavinii, O. Min. Sermones Dominicales et festivi ad fidem codicum recogniti, Costa, B., Frasson, L., Luisetto, I., Marangon P. (coll.) (1979), voll. I-III, Ed. Messaggero Padova, Padova.

Santo António de Lisboa (1970), Obras Completas, Introdução, tradução e notas H. Pinto Rema, O. F. M., voll. I-III, Ed. Restauração, Lisboa.

Dâmaso, M. S. (1755), Memorias dos beatos Jacobo de Bitecto, Ladislao de Gielnovo, Angelo de Clavasio e Joze' de Copertino, [n.i], Lisboa.

Secunda Synodus Diocesana ab Aloysio Pappacoda Episcopo Liciensi celebrata a Christi Nativitate Anno 1663, (1669), Success. Mascardi, Romae.

\section{Studi}

Alkofer, P. A. (2006), "José (Giuseppe) de Cupertino (apellido) Desa”, in Diccionario Enciclopédico de los Santos, Biografias y conceptos básicos del culto, voll. 1-3, 20032006, vol. II, G-O, Herder, Barcelona, 820-821.

Andrade, M. F. (2010), "Franciscanos", in J. E. Franco, J. A. Mourão, A. C. C. Gomes (dirs.), Dicionário Histórico das Ordens e Instituiçôes afins em Portugal, Gradiva Publicaçoes, Lisboa, 158-169.

Barthes, R. (1961), "Pour une psychosociologie de l'alimentation contemporaine", Annales ESC, XVI, 5: 977-986. 
Baschet, J. (2000), "I peccati capitali e le loro punizioni dell'iconografia medievale", in C. Casagrande, S. Vecchio, I sette vizi capitali. Storia dei peccati nel Medioevo, Einaudi, Torino: 225-260.

Belting, H. (2005), Bosch. Le Jardin des délices, Gallimard, Paris.

Bertelli, S., Centanni, M. (a cura di) (1995), Il gesto nel rito e nel cerimoniale dal mondo antico ad oggi, Ponte alle Grazie, Firenze.

Blanco, M. (2002), "Cultura”, in E. Caroli (a cura di), Dizionario Antoniano. Dottrina e spiritualità dei sermoni di Sant'Antonio, Ed. Messaggero, Padova, 127-147.

Bolzoni, L. (1995), La stanza della memoria. Modelli letterari e iconografici nell'età della stampa, Torino, Einaudi.

Bossy, J. (1998), "Aritmetica morale: sette peccati in dieci comandamenti, Idem, Dalla comunità all'individuo. Per una storia sociale dei sacramenti nell'Europa moderna, Einaudi, Torino, (1. ${ }^{\text {a }}$ ed. 1988), 87-116.

Braga, I. D., Braga, P. D. (2015), "Fazer a História dos animais”, in I. D. Braga, P. D Braga (coord.), Animais e Companhia na História de Portugal, Círculo de Leitores, Lisboa, 13-31.

Braga, I. D. (2015), “Carne e peixe: uma hierarquia de consumos alimentares”, in I. D. Braga, P. D. Braga (coord.), Animais e Companhia na História de Portugal, cit., 35-86.

Brambilla, E. (2000), Alle origini del Sant'Uffizio. Penitenza, confessione e giustizia spirituale dal medioevo al XVI secolo, Il Mulino, Bologna.

Caeiro, F. D. G. (1982), "Fonti portoghesi della formazione culturale di Sant'Antonio”, in A. Poppi (a cura di), Le fonti e la teologia dei sermoni antoniani, Ed. Messaggero Padova, Padova, 145-169.

Caeiro, F. D. G. (1981), "Fontes portugueses da formação cultural do Santo”, Itinerarium 27: 136-164.

Campanelli, M. (2013), "I conventi dei Minori Conventuali in Puglia nell'età di S. Giuseppe da Copertino (1603-1663)”, L'Idomeneo 15: 17-27.

Campanelli, M., (2004), "San Giuseppe da Copertino (1603-1663) e gli 'attori sociali' del processo neritino”, Studi Salentini LXXXI: 253-271.

Campos, J. T. (1990), Dicionário de Santos Hagiológico-Iconográfico, Lello \& Irmão, Porto.

Cargnoni, C. (2002), "Lussuria”, in E. Caroli (a cura di), Dizionario Antoniano, cit.: 431448.

Cargnoni, C. (2002), "Vizi”, in E. Caroli (a cura di), Dizionario Antoniano, cit.: 918-986.

Carvalho, J. R. (2011), “As sexualidades”, in J. Mattoso (ed.), História da vida privada em Portugal. A Idade Moderna, Temas e Debates, Círculo de Leitores, Lisboa, 96-129.

Coelho, M. H. da C. (1996), "Santo António de Lisboa em Santa Cruz de Coimbra”, in Congresso Internacional Pensamento e Testemunho, $8^{\circ}$ centénario do nascimento de Santo António, voll. I-II, Universidade Católica Portuguesa-Família Franciscana Portuguesa, Braga, vol. I, 179-205.

D’Ambrosio A., Spedicato, M. (1998), Cibo e clausura. Regimi alimentari e patrimoni monastici nel mezzogiorno moderno (sec. XVII-XIX), Cacucci, Bari. 
«Et il fuoco fa che la pignata mandi sopra la schiuma»: quando la lussuria sta in cucina?

Santi francescani italo-lusitani e disciplina sociale tra medioevo e prima etá moderna

Dalli Regoli, G. (2000), Il gesto e la mano: convenzione e invenzione nel linguaggio figurativo fra Medioevo e Rinascimento, Leo Olschki, Città di Castello.

De Boer, W. (2004), La conquista dell'anima. Fede, disciplina e ordine pubblico nella Milano della Controriforma, Einaudi, Torino, (2001, 1. ${ }^{\text {a }}$ ed.), 21-34.

Delumeau, J. (1978), Le péché et la peur: la culpabilisation en Occident (XIIIe-XVIIIe siècles), Fayard, Paris.

De Luca, F. (2000), Biblioteche monastiche in Puglia nel Cinquecento: Terra d'Otranto, vol. II, Conte Editore, Lecce.

Del Re, N. (1965), "Giuseppe da Copertino", in Bibliotheca Sanctorum, vol. VI, Istituto Giovanni XXIII Pontificia Università Lateranense, Roma, coll. 1300-1303.

Dias Barata, P. (2014), "De Spiritu Gastrimargiae. Distopia alimentar e gula na representação do inferno na tradição moral ocidental”, in C. Soares, I. Coutinho de Macedo (coord.), Ensaios sobre Património Alimentar luso-brasileiro, AnnablumeUniversidade Coimbra, São Paulo-Coimbra, 71-88.

Douglas, M. (1972), “Deciphering a Meal”, Daedalus 101.1: 61-81.

Fernandes, M. L. C. (2001), "Ignorância e confissão nas primeiras décadas do século XVII em Portugal”, in Estudos em homenagem a João Francisco Marques, vol. I, Porto, Universidade de Porto, 426-438.

Fernandes, M.L.C.(1995), "Do manual de confessores ao guia de penitentes. Orientações e caminhos da confissão no Portugal pós-Trento”, Via Spiritus 2: 47-65.

Fiorelli, V. (2013), "Costruire i santi e disciplinare i culti al tempo di S. Giuseppe da Copertino”, L'Idomeneo 15: 41-50.

Flandrin, J. L. (1981), Le sexe et l'Occident. Evolution des attitudes et des comportements, Seuil, Paris.

Frugoni, C. (2010), La voce delle immagini. Pillole iconografiche dal Medioevo, Einaudi, Torino.

Gardin, G. A. (1982), "Lussuria e castità in alcuni «Sermones» di S. Antonio”, Il Santo 22: 701-719.

Gentilcore, D. (2015), "Holy Food: Spiritual and Bodily Health", Idem, Food and Health in Early Modern Europe: Diet, Medicine and Society (1450-1800), Bloomsbury Publishing, London New York, 95-114.

Gentilcore, D. (2004), "Fra Giuseppe da Copertino, guaritore: testimonianze contemporanee", Studi Salentini LXXXI: 273-296.

Ginzburg,C.(1986), "Tiziano Ovidio e i codici della figurazione erotica nel Cinquecento”, in Idem, Miti emblemi, spie. Morfologia e storia, Einaudi, Torino, 133-157.

Gotor, M. (2004), Chiesa e santità nell'Italia moderna, Laterza, Roma-Bari.

Gouveia, J. R. (2015), A quarta porta do Inferno. A vigilância e disciplinamento da luxúria clerical no espaço luso-americano (1640-1750), Chiado Editora, Portugal-BrasilAngola-Cabo Verde.

Gouveia, J. (2011), O sagrado e o profano em choque no confessionário. O delito de solicitação no tribunal da Inquisição. Portugal 1551-1700, Palimage, Coimbra.

Lévi-Strauss, C. (2006), "Pequeno tratado de etnologia culinária”, in C. Lévi-Strauss, 
A origem dos modos à mesa. Mitológicas 3, Cisac Naify, São Paulo, [tr. B. PerroneMoisés; 1. ${ }^{\mathrm{a}}$ ed. 1968], 425-448.

Lévi-Strauss, C. (1966), Antropologia Strutturale, Milano, Il Saggiatore.

Mancino, M. (2000), Licentia confitendi. Selezione e controllo dei confessori a Napoli in età Moderna, Edizioni di Storia e Letteratura, Roma.

Marques, J. F. (2000), “Confissão”, in C. Moreira Azevedo (Dir.), Dicionário de História Religiosa de Portugal, vol. A-C, Círculos de Leitores, Lisboa, 445-459.

Marques, J. F. (2000), "Pregação”, in História Religiosa de Portugal (ed. C. M. de Azevedo), Círculo de Leitores, Lisboa, vol. II, 393-447.

Miele, M. (1997), “Confessione, confessori e penitenti nei sinodi di area napoletana della seconda metà del Cinquecento", in B. Ulianich (a cura di), Ricerche sulla confessione dei peccati a Napoli tra '500 e '600, Città del Sole, Napoli, 15-64.

Montanari, M. (2012), Gusti del Medioevo. I prodotti, la cucina, la tavola, Laterza, RomaBari.

Montanari, M. (2006), Il cibo come cultura, Laterza, Roma-Bari.

Montanari, M. (1991), Nuovo Convivio: storia e cultura dei piaceri della tavola nell'età moderna, Laterza, Roma-Bari.

Moreira, A. M. (2000), "Franciscanos", in C. M. Azevedo (Dir.) Dicionário de História Religiosa de Portugal C-I, Círculo de Leitores, Lisboa, 273-280.

Mormando, F. (2011), Bernini His Life and His Rome, University of Chicago Press, Chicago.

Nestola P. (2015), “S. Giuseppe da Copertino vs S. José de Cupertino: proposte storiografiche tra territorialità e universalità", in P. Nestola. (a cura di), Allegramente: quando Servire è un piacere. Miscellanea in memoria di p. Antonio Fanuli C. M., Ed. Grifo, Lecce, 327-341.

Nestola, P. (2013), "S. Jozé de Cupertino: "santo dei voli” in Portogallo? Itinerari di ricerca tra letteratura, iconografia e rappresentazione sociale”, Lusitania Sacra 28: 95-118.

Nestola, P. (2010), “S. Giuseppe da Copertino, un patronato toponimico emblematico: da santo nella sua "terra" a civica insegna identitaria (1664-1858)", in M. Spedicato (a cura di), Santi patroni e identità civiche nel Salento moderno e contemporaneo, Edipan, Galatina, 19-64.

Nestola, P. (2008), I grifoni della fede. Vescovi-inquisitori in Terra d'Otranto tra '500 e 600, [Prefazioni di M. Spedicato e J. P. Paiva], Congedo, Galatina.

Nestola, P. (2004), "La Santità al femminile: San Giuseppe da Copertino tra culto popolare e culto elitario", L'Idomeneo 6: 27-42.

Novi Chavarria, E. (2004), "San Giuseppe e i modelli di santità nel Mezzogiorno moderno”, Studi Salentini LXXXI: 215-231.

Nunes, J. R. (2014), “A reforma católica na diocese de Viseu (1552-1639)”, in A. Camões Gouveia, D. Sampaio Barbosa, J. P. Paiva (coords.), O Concílio de Trento em Portugal e nas suas conquistas. Olhares novos, CEHR, Universidade Católica Portuguesa, Lisboa, 59-78. 
«Et il fuoco fa che la pignata mandi sopra la schiuma»: quando la lussuria sta in cucina?

Santi francescani italo-lusitani e disciplina sociale tra medioevo e prima etá moderna

Paiva, J. P. (2013a), "Episcopato e predicazione nel Portogallo Moderno: forme di attuazione e di vigilanza”, in J. P. Paiva, Un episcopato vigile. Portogallo, secoli XVIXVIII, [trad. P. Nestola], Ed. Grifo, Lecce, 217-258.

Paiva, J. P. (2013b), "Una dottrina per credere e obbedire, in J. P. Paiva, Un episcopato vigile. Portogallo, Secoli XVI-XVIII, [trad. P. Nestola], Ed. Grifo, Lecce, 259-302.

Paiva, J. P. (2011), "Uma doutrina para acreditar e obedecer”, in J. P. Paiva, Baluartes da fé e da disciplina. O enlace entre a Inquisição e os bispos em Portugal (1536-1750), Imprensa da Universidade de Coimbra, Coimbra, 261-309.

Paiva, J. P. (2009), “Episcopado e pregação no Portugal Moderno: forma de actuação e de vigilancia”, Via Spiritus 16: 7-42.

Paiva,J.P.(2006), Os Bispos de Portugal e do Império, 1495-1777, Imprensa da Universidade, Coimbra.

Paiva, J. P. (2000), “Constituições Diocesanas”, in C. M. de Azevedo (ed.), Dicionário de História religiosa de Portugal, vol. C-I, Círculo de Leitores, Lisboa, 9-15.

Palomo, F. (2006), A Contra-Reforma em Portugal 1540-1700, Livros Horizonte, Lisboa, 41-50.

Pinto Rema, H. (2002), "Sermoni”, in E. Caroli (a cura di), Dizionario Antoniano, cit.: 723-738.

Prosperi, A. (1996), Tribunali della coscienza. Inquisitori, confessori, missionari, Einaudi, Torino.

Renoux, C. (1993), “Une source de l'histoire de la mystique moderne revisitée: les procès de canonisation", Mélanges de l'Ecole Française de Rome. Italie et Méditerranée 105.1: 177-217.

Rigon, A. (2002), Dal Libro alla folla. Antonio da Padova e il francescanesimo medievale, Viella, Roma.

Rodrigues, D. (2007), Grão Vasco, Alêtheia, Lisboa.

Rodrigues, D. (1992), "Vasco Fernandes e a oficina de Viseu”, in F. Faria Paulino, Grão Vasco e a pintura europeia do Renascimento, Comissão Nacional Para as Comemorações dos Descobrimentos Portugueses, Lisboa, 144-151.

Romeo, G. (1998), Esorcisti, confessori e sessualità femminile nell'Italia della Controriforma, Le Lettere, Firenze.

Romeo, G.(1997), Ricerche su confessione dei peccati e Inquisizione nell'Italia del Cinquecento, Istituto Italiano di Studi Filosofici, Napoli.

Rusconi, R. (2002), L'ordine dei peccati. La confessione tra Medioevo ed età Moderna, Il Mulino, Bologna.

Rusconi, R. (1981), "Predicatori e predicazione”, in C. Vivanti, (a cura di), Storia d'Italia, Annali 4, Intellettuali e potere, Einaudi, Torino, 951-1035.

Sangalli, M. (2007), "La formación del clero en la edad moderna. De Roma, a Italia a Europa”, Manuscrits 25: 108-111.

Sallmann, J. M. (1994), Naples et ses saints à l'âge baroque (1540-1750), PUF, Paris.

Santos, L. R. (1945), Santo António na Pintura Portuguesa do século XVI, Editorial Ática, Lisboa. 
Sarti, R. (2012), "Melhor o cozinheiro? Um percurso sobre a dimensão de gênero da preparação da comida (Europa occidental, séculos XVI-XIX)”, Cadernos Pagu 39: $87-158$.

Sarti, R. (2003), Vita di casa: abitare, mangiare vestire nell'Europa moderna, Laterza, Roma-Bari.

Schmitt, J. C. (1984), “Gestures”, History and Antropology I.1: 1-28.

Silva, C. (2010), "Franciscanos Conventuais”, in J. Eduardo Franco, J. Augusto Mourão, A. C. da Costa Gomes (dirs.), Dicionário Histórico, cit.: 169-173.

Sodano, G. (2004), "Il processo di canonizzazione pugliese di San Giuseppe da Copertino: aspetti generali e peculiarità”, Studi Salentini LXXXI: 233-251.

Spedicato, M. (2015), "La fabbrica di un santo. I processi di canonizzazione di fra Giuseppe Desa da Copertino", in P. Nestola, (a cura di), Allegramente, cit.: 315326.

Spedicato, M. (2009a), "Le virtù eroiche di un santo del Salento. San Giuseppe da Copertino (1603-1663)", in B. Pellegrino (a cura di), Ordini religiosi, santi e culti tra Europa, Mediterraneo e Nuovo Mondo, Atti del V Convegno internazionale AISSCA, Lecce 3-6 maggio 2003, Congedo, Galatina, vol. II, 495-512.

Spedicato, M. (2009b), "Francescanesimo e adozione dei santi patroni nel Salento moderno e contemporaneo", in M. Spedicato, Simboli identitari. Studi sui santi patroni del Salento moderno, EdiPan, Galatina, 17-31.

Teixeira, V. (2015), "Introdução", in J. E. Franco (Dir.), Dicionário Família Franciscana em Portugal. Ordens e outras formas de vida consagrada, Lucerna, Cascais, 7-53.

The Saint of the Child Jesus, Saint Anthony. Art and History (1995), Museu Nacional de Arte Antiga, Lisboa.

Turrini, M. (1991), La coscienza e le leggi. Morale e diritto nei testi per la confessione della prima età moderna, Il Mulino, Bologna, 189-241.

\section{Siti web consultati}

www.santantonio.org/portale/sermoni 